\title{
Inclusion Exclusion Criterion Character Result in Standard Format
}

National Cancer Institute

\section{Source}

National Cancer Institute. Inclusion Exclusion Criterion Character Result in Standard

Format. NCI Thesaurus. Code C83301.

The standard character or string for representation and reporting of inclusion exclusion criterion data. 